\title{
Relationships Between Horizontal Interactions and Functional Architecture in Cat Striate Cortex as Revealed by Cross-Correlation Analysis
}

\author{
Daniel Y. Ts'o, Charles D. Gilbert, and Torsten N. Wiesel \\ Laboratory of Neurobiology, The Rockefeller University, New York, New York 10021
}

Anatomical studies in the visual cortex have shown the presence of long-range horizontal connections with clustered axonal collaterals, suggesting interactions over distances of several millimeters. We used cross-correlation analysis in cat striate cortex to detect interactions between cells over comparable distances. Using one cell as a reference, we recorded from other cells with a second electrode at varying distances and looked for correlated firing between the two recording sites. This technique allowed us to combine a physiological measure of the strength and type of connection between cells with a characterization of their receptive field properties. The observed interactions were excitatory, and extended over horizontal distances of several millimeters. Furthermore, the interactions were between orientation columns of like specificity, resulting in a waxing and waning in the strength of interaction as the electrodes passed through different orientation columns. We studied relationships between strength of correlation and other receptive field properties and found a tendency for facilitatory interactions between cells sharing the same eye preference. A large proportion of our correlations was due to common input. This feature, and the similarity of interactions between cells in the same column with the reference cell, suggest a high degree of interconnectivity between and within the columns. As the distance between the two electrodes increased, the overlap of the receptive fields of the cells participating in the interactions gradually diminished. At the furthest distances recorded, the cell pairs had nonoverlapping receptive fields separated by several degrees. The distribution and range of these interactions corresponded to the clustering and extent of the horizontal connections observed anatomically.

The cat visual cortex has a columnar structure, where cells having certain receptive field properties in common are distributed in vertical columns running from pia to white matter (Hubel and Wiesel, 1962). These properties, including orientation and ocular dominance, are repeated in a regular fashion. Within a distance of $700-800 \mu \mathrm{m}$ parallel to the cortical surface, one encounters a complete set of orientation or ocular dominance columns. This unit is termed the hypercolumn (Hubel and Wiesel, 1974). Cells in a hypercolumn have receptive fields positioned in a restricted region of visual space; after a traversal of two hypercolumns, the receptive fields have shifted so that they do not overlap with those of the previous site. This ar-

\footnotetext{
Received Aug. 12, 1985; revised Oct. 7, 1985; accepted Oct. 9, 1985.

We thank Jurgen Bolz, Roberta Brinton, Amiram Grinvald, Larry Katz, and Barbara McGuire for their comments on the manuscript; Joyce Powzyk for illustrations; Peter Pierce for photography; and Julie Dollinger and Ann Reardon for histology. The work was supported by NIH Grants EY05253 and EY04782 and NSF Grants BNS9318794 and BNS8351738.

Correspondence should be addressed to D. Y. Ts'o, Laboratory of Neurobiology, The Rockefeller University, 1230 York Avenue, New York, NY 10021.

Copyright @ 1986 Society for Neuroscience $0270-6474 / 86 / 041160-11 \$ 02.00 / 0$
}

rangement allows each part of the visual field to be analyzed in terms of all orientations and by both eyes.

To maintain the visuotopic order of the cortex one would expect that connections would be fairly restricted in their horizontal extent. This view was consistent with Golgi studies that showed that connections ran primarily perpendicular to the cortical surface, across the layers (Lorente de No, 1933). However, subsequent anatomical studies have demonstrated the unexpected presence of horizontal intrinsic connections spanning several millimeters (Fisken et al., 1975; Gilbert and Wiesel, 1979; Rockland et al., 1982). Furthermore, these connections were shown to be nonuniformly distributed. Cells labeled by intracellular HRP had long-ranging axons with collaterals arranged in discrete clusters, suggestive of connections between the cortical columns (Gilbert and Wiesel, 1979, 1983; Martin and Whitteridge, 1984).

The role of the clustered horizontal connections is not known, but it has been proposed that they may serve to connect columns of the same orientation (Gilbert and Wiesel, 1981, 1983; Mitchison and Crick, 1982). We were therefore interested in determining whether there was any consistent relationship between the horizontal connections and the cortical columns. The interaction could be excitatory or inhibitory, and could influence columns of similar or different specificity. To resolve these issues, we have used cross-correlation analysis, which provides a physiological measure of the type and strength of interactions between neurons. These methods and their interpretation were introduced to neurophysiology by Perkel et al. (1967a, b). Toyama et al. (1981a, b) used cross-correlation methods to investigate vertical and local connections in the visual cortex and explore interactions between cell types. Michalski et al. (1983) examined the types of interactions in visual cortex occurring between neighboring cells and between sites separated by distances up to $1 \mathrm{~mm}$. Our experiments show the dependency of these interactions on receptive field properties and demonstrate the interactions over longer distances, comparable to the extent of the horizontal connections.

\section{Materials and Methods}

Cats were initially anesthetized with ketamine $\mathrm{HCl}(20 \mathrm{mg} / \mathrm{kg}$, i.m.) followed by sodium pentothal $(20 \mathrm{mg} / \mathrm{kg}$, i.v., supplemented by further injections as needed). The electrocardiogram, electroencephalogram (EEG), temperature, and expired $\mathrm{CO}_{2}$ were monitored. The animal was then intubated with an endotracheal tube or cannulated through a tracheotomy, paralyzed with succinylcholine $(10 \mathrm{mg} / \mathrm{kg} / \mathrm{hr})$, and artificially respirated. The EEG was monitored and sodium pentothal was given i.p. continuously at a rate to assure that the animal was asleep (1-3 mg $\mathrm{kg} / \mathrm{hr}$ ). The nictitating membranes were retracted with $10 \%$ phenylephrine, and the pupils were dilated with $1 \%$ atropine. A retinoscope was used to determine the appropriate contact lens to focus the eyes on a tangent screen $1.5 \mathrm{~m}$ from the animal. The positions of the area 
A

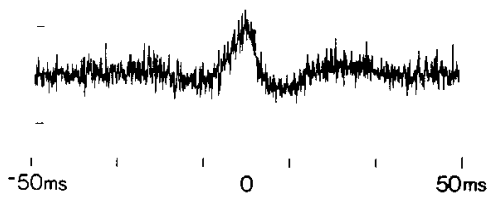

$-\mathrm{B}$

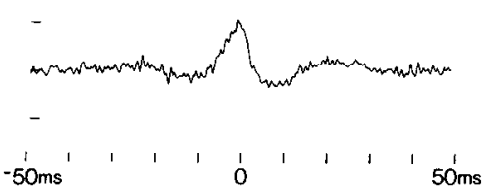

$-\mathrm{C}$

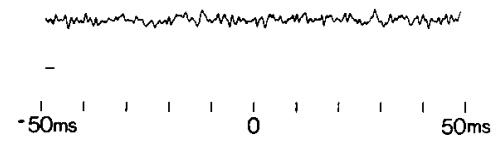

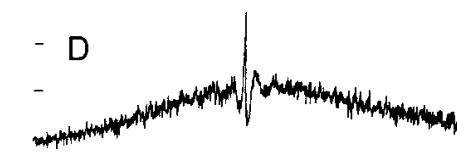

$400 \mathrm{~ms}$

0

$400 \mathrm{~ms}$
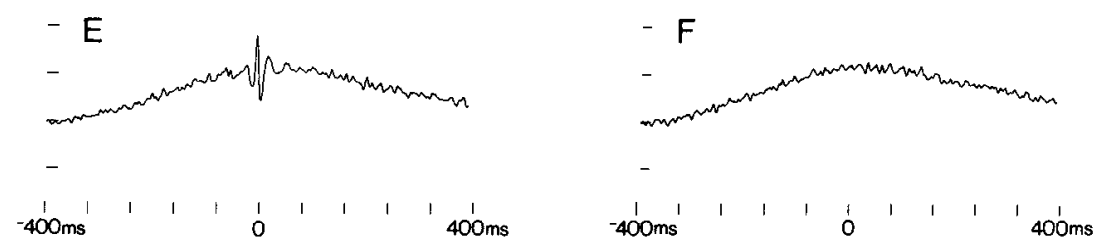

Figure 1. Stimulus coordination artifact. $A$ and $D$, Raw correlograms obtained from a pair of cells with similar receptive field properties. $C$ and $F$. Shuffled correlograms obtained from the same cell pair. $A-C$, Correlograms collected with a $\perp 50$ msec window; $D-F$, collected with a \pm 400 msec window. $B, C, E$, and $F$ have been processed by a low-pass filter to remove noise. Here and in subsequent figures, each tick mark on the ordinate represents an integral multiple of the baseline level of correlation. The number of spikes collected were $N_{a}=16,355, N_{b}=11,950$. The mean baseline value for $A$ was 29.1 spikes/bin; $D, 124.3$ spikes/bin.

centralis of each eye were plotted on the screen with the aid of a fundus camera (Nikon).

A hole of about $3 \mathrm{~mm}$ in diameter was made in the skull at HorsleyClarke posterior $0-5 \mathrm{~mm}$ near the midline and the dura, and, on occasion, the pia were opened. After placement of the two electrodes in the cortex, the hole was filled with agar to reduce pulsations. In most experiments, etched lacquer-coated tungsten wire electrodes were used for the recordings (Hubel, 1957). For the pharmacological stimulation of cortical cells we used double-barrel glass electrodes (Kasser and Cheney, 1983), in which one barrel contained an etched tungsten wire for recording and the other contained $0.2 \mathrm{M} \mathrm{D}, \mathrm{L}$-homocysteic acid (DLH), pII 7.5, a glutamate analog. DLH was ejected with currents ranging from 5 to $25 n \mathrm{~A}$.

Electrolytic lesions were made to mark positions along the penetrations for subsequent histological reconstruction. Lesions of approximately $100 \mu \mathrm{m}$ in diameter were made by passing $3 \mu \mathrm{A}$ of current through the electrode for $3 \mathrm{sec}$. After the experiment was finished, the animal was perfused with $4 \%$ formaldehyde. Blocks of tissue containing the electrode tracks were sectioned on a freezing microtome (American Optical Co.) to $30 \mu \mathrm{m}$ thickness. These sections were mounted on gelatinized slides and stained with cresyl violet. Lesions were located and the laminar position determined by the criteria of Otsuka and Hassler (1962). The electrode tracks and lesions were reconstructed using a camera lucida-equipped microscope (Zeiss).

Each electrode was advanced with a microstepper motor (Ardel-Kinematic). The receptive field position and properties of encountered single units were studied with slits of light from a hand-held projector. The orientation preference, directionality, ocular dominance, receptive field size and position, extent of end inhibition, edge preference, and response to stationary light flashes for each unit were measured. Cells were classified into ocular dominance groups 1-7 according to Hubel and Wiesel (1962). Cells were also classified by the strength of directionality into groups $0-4$. Directionality group 0 cells exhibited no preference for either direction of stimulus motion orthogonal to the optimal orientation; group 4 cells responded exclusively to a particular direction of stimulus motion. Likewise for end inhibition, cells were placed into end-inhibition groups $0-4$; group 0 cells had no reduction in response to increasingly longer slits of light, group 4 cells had a complete elimination of their response to long slits. A microprocessor-controlled optic bench was used to provide light stimuli for responsc histograms and correlograms. The two extracellularly recorded signals were amplified and fed into two window discriminators that provided digital pulse outputs. The threshold levels of the window discriminators were constantly monitored for proper triggering to a single unit. The two digital pulse outputs were processed in real-time by a microprocessor (DEC PDP 11/23) to provide online computation and display of stimulus histograms, autocorrelograms, and cross-correlograms.

\section{Cross-correlation analysis}

Raw cross-correlograms were computed by accumulating a histogram of time differences between the occurrence of spikes in the two recorded cells. The left group of bins in the histogram represents the negative times, when the second cell fired prior to the first. The center, or zero, bin of the histogram accumulates the number of instances when the two cells fired precisely together. The right group of bins, the positive times, account for the various time intervals that the first cell fired prior to the second cell. The temporal resolution of the real-time correlator was $100 \mu \mathrm{sec}$, and cross-correlograms were accumulated with $\pm 50 \mathrm{msec}$. To be able to detect longer-lasting synaptic events we also computed the cross-correlograms for a $\pm 400 \mathrm{msec}$ window. However, none of the interactions observed in our study extended beyond the $\pm 50 \mathrm{msec}$ window (see Fig. 1), so only the correlograms collected at the short time window were used in subsequent analyses. A minimum of 3000 spikes was collected from each cell to insure an adequate signal-to-noise ratio in the correlograms. To collect a sufficient number of spikes from each cell in a reasonable amount of time, each cell was stimulated with an optimal light stimulus. In cases where the two cells had quite different receptive field properties and/or position, two different light slits were used to provide and optimal stimulus to each cell. The time required to collect a minimum of 3000 spikes from each cell varied between 10 and $30 \mathrm{~min}$.

There are previous discussions in the literature that provide guidelines to interpret cross-correlograms and to infer details of the underlying circuitry (Bryant et al., 1972; Moore et al., 1970, Perkel et al., 1967a, b). Before one can begin to interpret the cross-correlograms, it is important to be certain that any stimulus coordination artifact will not affect the interpretation. The artifact is due to the elevation of the average firing rates of the cells by the visual stimulus. This synchronous elevation in firing rates increases the random, baseline probability that the two cells will fire close to the same time and can affect the resultant cross-correlogram. To control for the stimulus coordination artifact we first isolated the artifact itself by computing the shuffled correlogram, correlating one spike train with the other spike train shifted in time by one visual stimulus period. This shuffled correlogram is then subtracted from the raw correlogram, thereby removing the component of the raw correlogram due to stimulus coordination. Both shuffle-adjusted and raw correlograms were available online, and all the correlograms presented have been shuffled-adjusted.

We observed that the stimulus coordination artifact does not usually influence the shape of the cross-correlogram collected at $\pm 50 \mathrm{msec}$ but does at $\pm 400 \mathrm{msec}$. An example of a raw correlogram is shown in Figure $1 A$ and $B$, and its companion shuffled correlogram in Figure $1 C$, both collected at $\pm 50 \mathrm{msec}$. Figure $1, D-F$, shows the same raw and shuffled correlograms collected at $\pm 400 \mathrm{msec}$. The effect of the stimulus coor- 


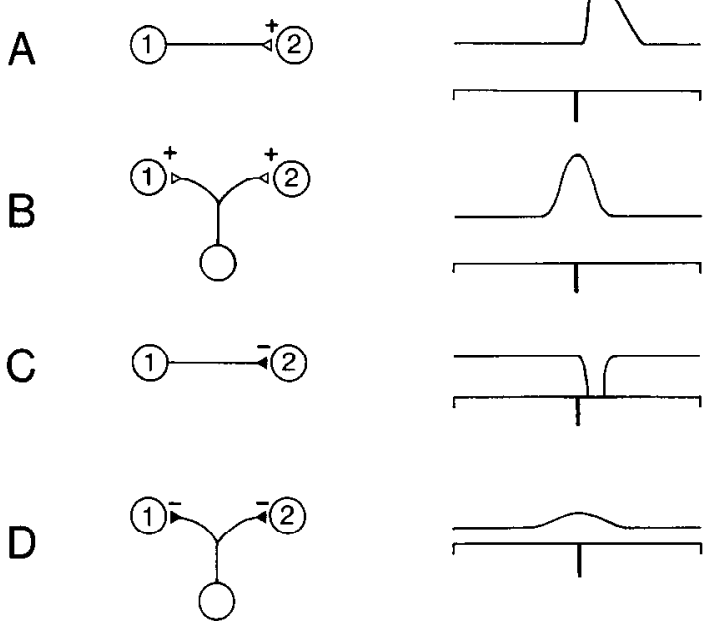

Figure 2. Idealized correlograms from four basic neuronal circuits. $A$, Direct excitation from cell 1 to cell 2 , producing an asymmetric positive peak, offset to one side of the zero time bin. $B$, Common excitation to cells 1 and 2 from a third cell, producing a positive peak, symmetric about zero. $C$, Direct inhibition from cell 1 to cell 2, producing a downward trough from baseline, offset to one side of zero. $D$, Common inhibition to cells 1 and 2 from a third cell, producing a small, broad positive peak, symmetric about zero. Adapted from Moore et al. (1970).

dination artifact in the \pm 50 msec correlogram is to raise its baseline by a constant amount, but it does not change the shape of the peaks. The stimulus coordination artifact (shown in Fig. $1, D-F$ ) manifests as a broad peak with a time course of several hundred milliseconds, in contrast to the much sharper peaks characteristic of neuronal interactions. Therefore, the two can be readily distinguished and separated in the raw correlogram. Factors such as the difference in position of the two receptive fields, which alone can yield a few hundred millisecond time difference between the appearance of the light stimulus in the two receptive fields, as well as fluctuations in the latency of response, would account for the few hundred millisecond time course of the stimulus coordination artifact. This situation contrasts with earlier cross-correlation studies in auditory cortex (Dickson and Gerstein, 1974), where auditory neurons fire with a fixed phase relationship to the auditory stimuli, resulting in stimulus coordination artifacts with similar temporal properties to the direct neuronal interactions under study.

In addition to stimulus coordination, cells with periodic and refractory firing behavior may also yield misleading features in the cross-correlogram. For example, side dips adjacent to the central cross-correlogram peak may be due to the refractory period of the cells involved. Autocorrelograms of each cell were accumulated to monitor these situations.

The basic patterns of neuronal interaction shown in a cross-correlogram are distinguished by the position of the peaks relative to the zero bin and the shape of the peaks. Simplified examples of neuronal circuitry and their expected resulting cross-correlograms are shown in Figure 2. Common input interactions yield peaks that usually straddle the zero bin and are often symmetrical (Fig. $2 B$ ). Delayed excitation peaks attributed to direct monosynaptic input should be shifted from the zero bin by a time that corresponds to the synaptic latency (Fig. $2 A$ ). These peaks are generally more asymmetric than a common excitation peak. Their shape is thought to be related to the EPSP and synaptic jitter (Gustafsson and McCrea, 1984; Kirkwood, 1979; Surmeier and Weinberg, 1985). Though it may be possible to detect multisynaptic connections in cross-correlograms, it is expected that these peaks would be broader and shallower than peaks due to monosynaptic connections. Delays in peaks may also be due to conduction latencies, but this effect is expected to be small for intrinsic connections. Inhibitory monosynaptic input appears as a valley in the correlogram, with a time shift corresponding to synaptic latency (Fig. $2 C$ ). Common inhibitory input generates a positive peak, which is expected to be broader and smaller than that seen with common excitation (Fig. 2D).

Because of differences in spike collecting conditions, such as the time period of collection and the average firing rates of the cells, the noise levels of the correlograms varied greatly and were sometimes high. To uncover features in the correlograms that were hidden by high noise levels, the correlograms were routinely processed by a linear low-pass filter program that computes a weighted average of each nine bins. All cross-correlograms were normalized by the baseline to allow a more accurate accessment of the relative strengths of the revealed neuronal interactions. However, the differences in noise level between correlograms remain. The $y$-axis of each normalized correlogram corresponds to the strength of the correlation as multiples of the baseline (random) correlation. The baseline of each $\pm 50 \mathrm{msec}$ correlogram was determined by averaging the bin values in the outermost $5 \mathrm{msec}$ bins, where no correlation peaks ever appeared. We derived a numerical index of the strength of interaction from each correlogram for use in summary graphs and histograms. This strength index was computed by estimating the energy of the correlogram above the noise (baseline) level. The formula for the strength index used (after low-pass filtering) was

$$
S I=\left[\Sigma\left(x_{i}-s_{i}\right)^{2}-\Sigma\left(b_{i}-s_{i}\right)^{2}\right]^{1 / 2} / \bar{b}
$$

where $x_{i}$ is the raw correlogram, $s_{i}$ the shuffled correlogram, and $b_{i}$ the baseline of the raw correlogram. Note that since our shuffled correlograms were flat,

$$
s_{i} \approx b_{i} \text { and } \bar{s} \approx b
$$

\section{Results}

Results were compiled from data and correlograms (see Materials and Methods) of 115 cell pairs from nine penetrations in nine cats. Our first observation was that correlated firing usually depended on matching receptive field properties, while cell pairs with dissimilar receptive field properties tended to exhibit no correlated firing. An example of this finding is shown in Figure 3. In this instance two electrodes were placed in the superficial layers of area 17 at a separation of $700 \mu \mathrm{m}$. Single units were
Figure 3. Correlograms obtained from two cell pairs. $A$, The cell pair had similar receptive properties: The first cell had an orientation preference of $120^{\circ}$, directional preference to the right and an ocular dominance group of 2 , the second cell had identical orientation and direction preference and an ocular dominance group of 3 . $B$, The first cell was the same cell as the first cell in $A$. The second cell had different receptive ficld properties: an orientation preference of $20^{\circ}$, upward directionality, and an ocular dominance of 5 .
A

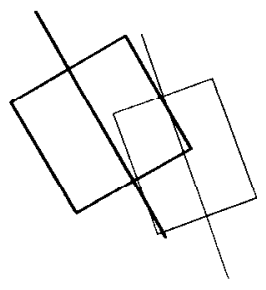

B

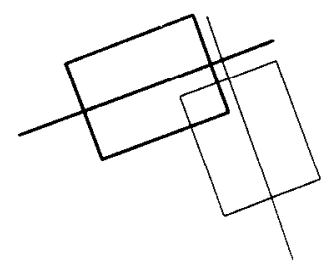

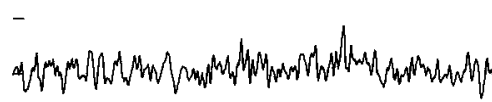

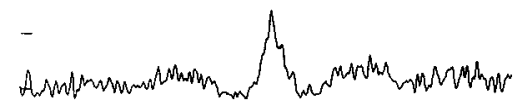

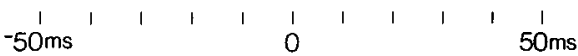

-

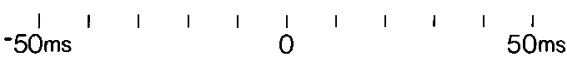



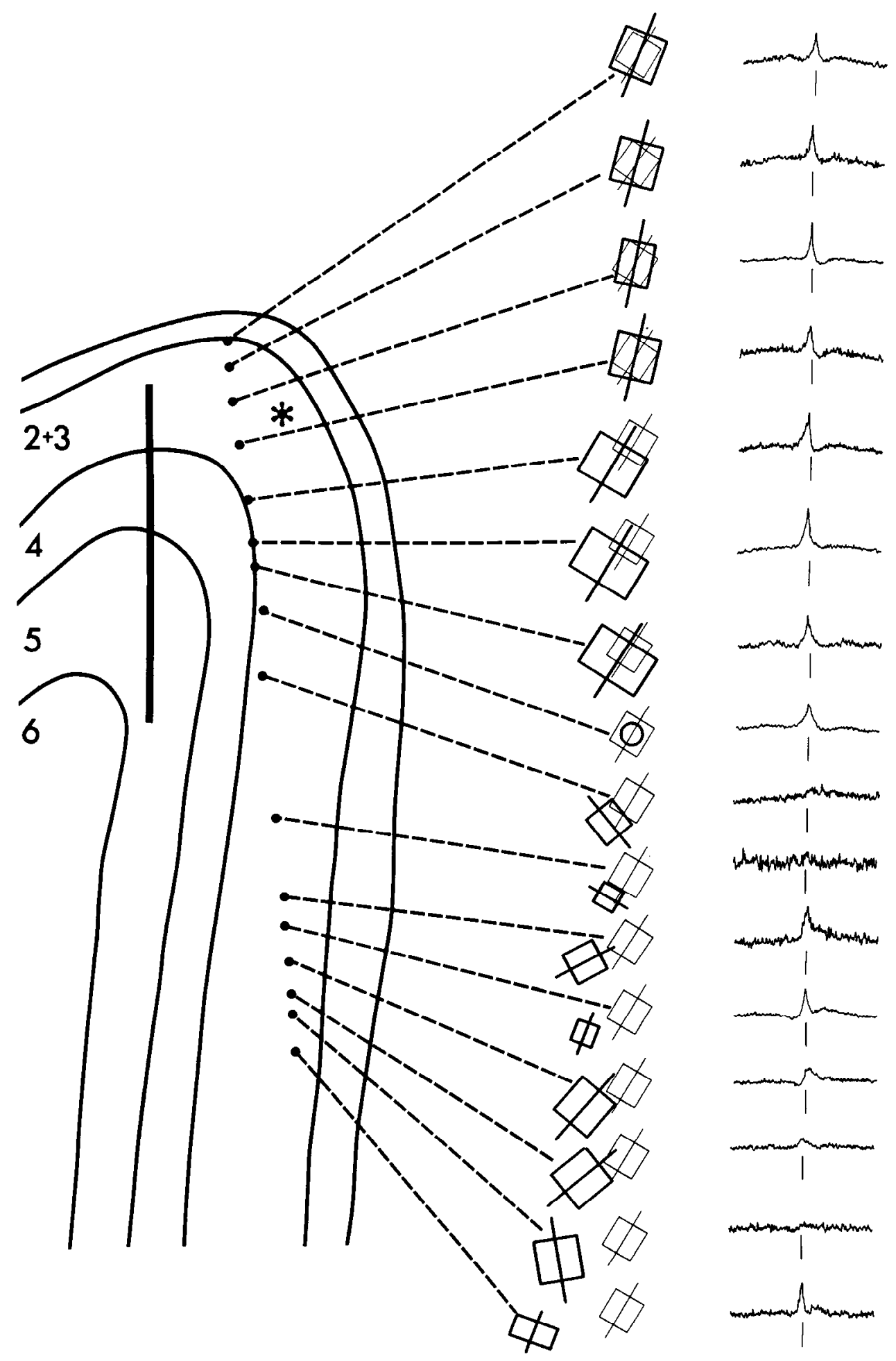
with correlograms between a reference cell and various test cells. The reference recording site is shown as the asterisk and had an orientation preference of $60^{\circ}$. Its receptive field map and position are shown in each pair with light lines.The size of the reference cell receptive field was $1^{\circ} \times 1^{\circ}$. Each test cell recorded in the second electrode has its receptive field shown in bold lines. The shuffled-adjusted, noise-filtered, and normalized correlogram obtained from each test cell (with respect to the reference cell) is shown at the far right. The cell displayed with a circular receptive field had no detectable orientation preference. The correlograms were collected with a $\pm 50 \mathrm{msec}$ window, and the bottom vertical tick mark midway between each correlogram denotes the zero time bin. Scale bar, 1 $\mathrm{mm}$.

isolated with each electrode, receptive fields characterized, and correlograms taken. The first cell had an orientation tuning of $120^{\circ}$, directional preference to the right, and an ocular dominance group of 2 . The second cell had similar orientation and directional preferences and a similar ocular dominance. Figure $3 A$ is the correlogram obtained from this cell pair. This correlogram has a sharp positive peak that straddled the zero time bin, indicating a common excitatory influence. The second electrode was then moved to isolate a cell with quite different receptive field properties while the first electrode was kept fixed. This third cell was separated from the first cell by $1 \mathrm{~mm}$ and had an orientation preference of $20^{\circ}$, upward directionality, and an ocular dominance of group 5 . Figure $3 B$ is the correlogram obtained between the first and third cells. The correlogram was flat, indicating there was no observable interaction between these two cells with differing receptive field properties.

To explore systematically the relationship between the longrange horizontal connections and receptive field properties, we made electrode penetrations that were parallel to the surface of the cortex, down the medial bank of the lateral gyrus. This approach enabled us to stay in a particular layer for several millimeters, and record from a series of cells with receptive 
Figure 5. Medial bank penetration similar to Figure 3. Note tendency for the diminishing strength of the peaked correlograms with increascs in distance between cells along the medial bank penetration and the reference cell. Also note the large difference in receptive field positions between the recording sites furthest apart.

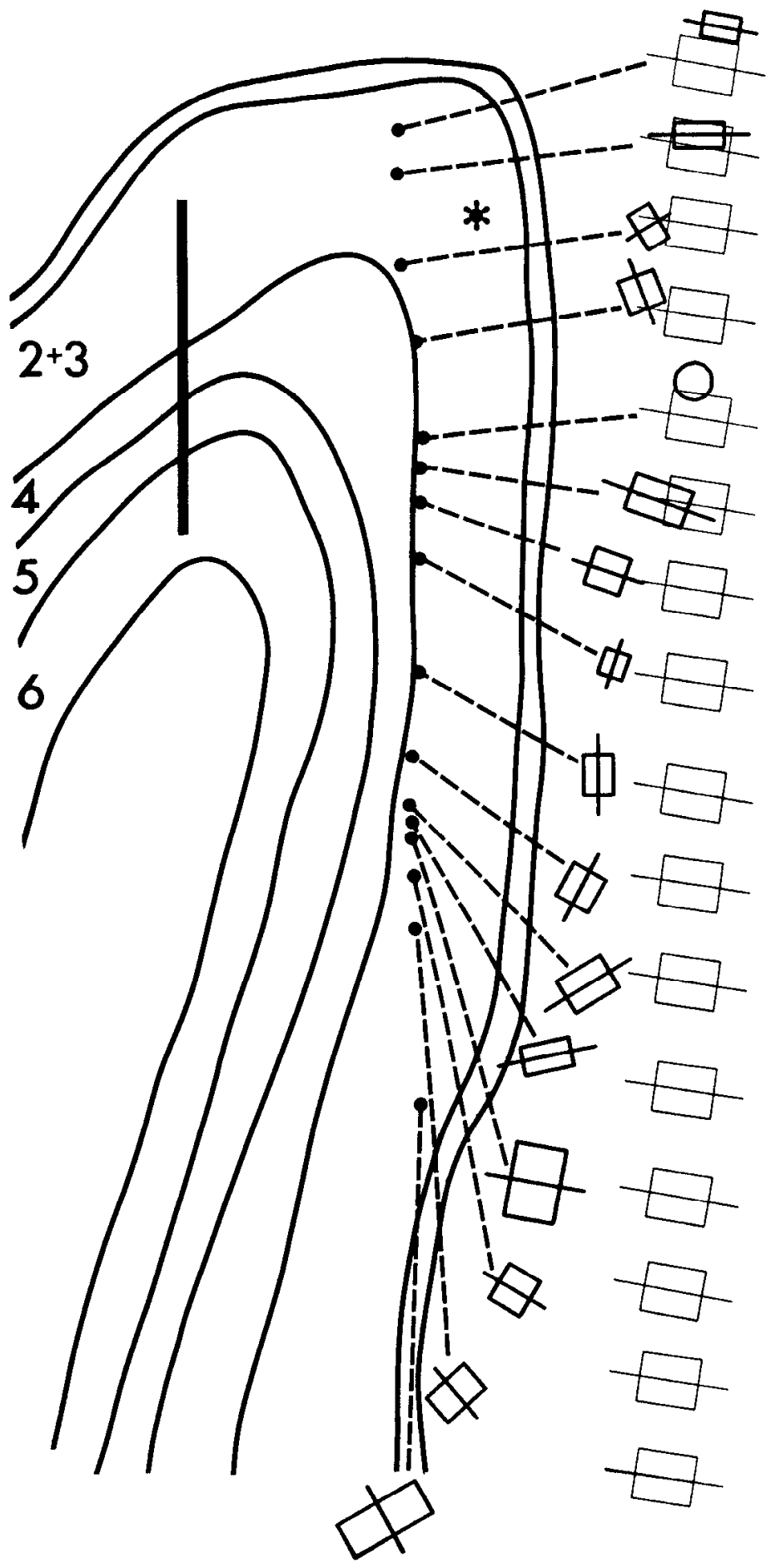

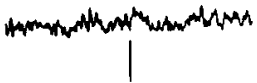
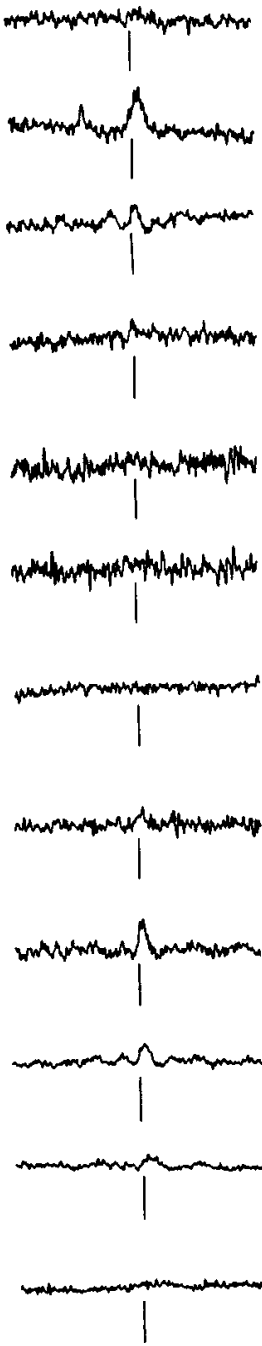

fields displaced from the midline, parallel to the horizontal meridian. Along the penetration we encountered a successsion of different orientations and ocular dominance shifts. Another electrode isolated a single unit that was used as a reference cell throughout the experiment. Using this strategy, we were able to study the neuronal interactions between a reference site in a single cortical column and other cells in other columns at varying horizontal distances and with a range of receptive field properties. To limit our study to the horizontal, as opposed to interlaminar, connectivity, we restricted our penetrations to layer $2+3$.

The dependency of correlated firing on matching receptive field properties was replicated over the length of our medial bank penetrations, covering several millimeters. Figure 4 shows an example of such a penetration. The reference cell had an orientation of $60^{\circ}$. The cells initially encountered by the second electrode had orientation preferences that were similar to the reference cell. For example, the first cell isolated had an orientation of $70^{\circ}$. The resultant cross-correlogram was sharply peaked. Throughout the penetration peaked correlograms were only found with groups of cells having similar orientation preferences, interposed by regions of cells that had flat correlograms. The correlograms had robust peaks for orientation differences up to about $30^{\circ}$. The waxing and waning of correlated firing extended for the entire $2 \mathrm{~mm}$ length of the electrode penetration. Towards the end of the penetration we still encountered peaked 


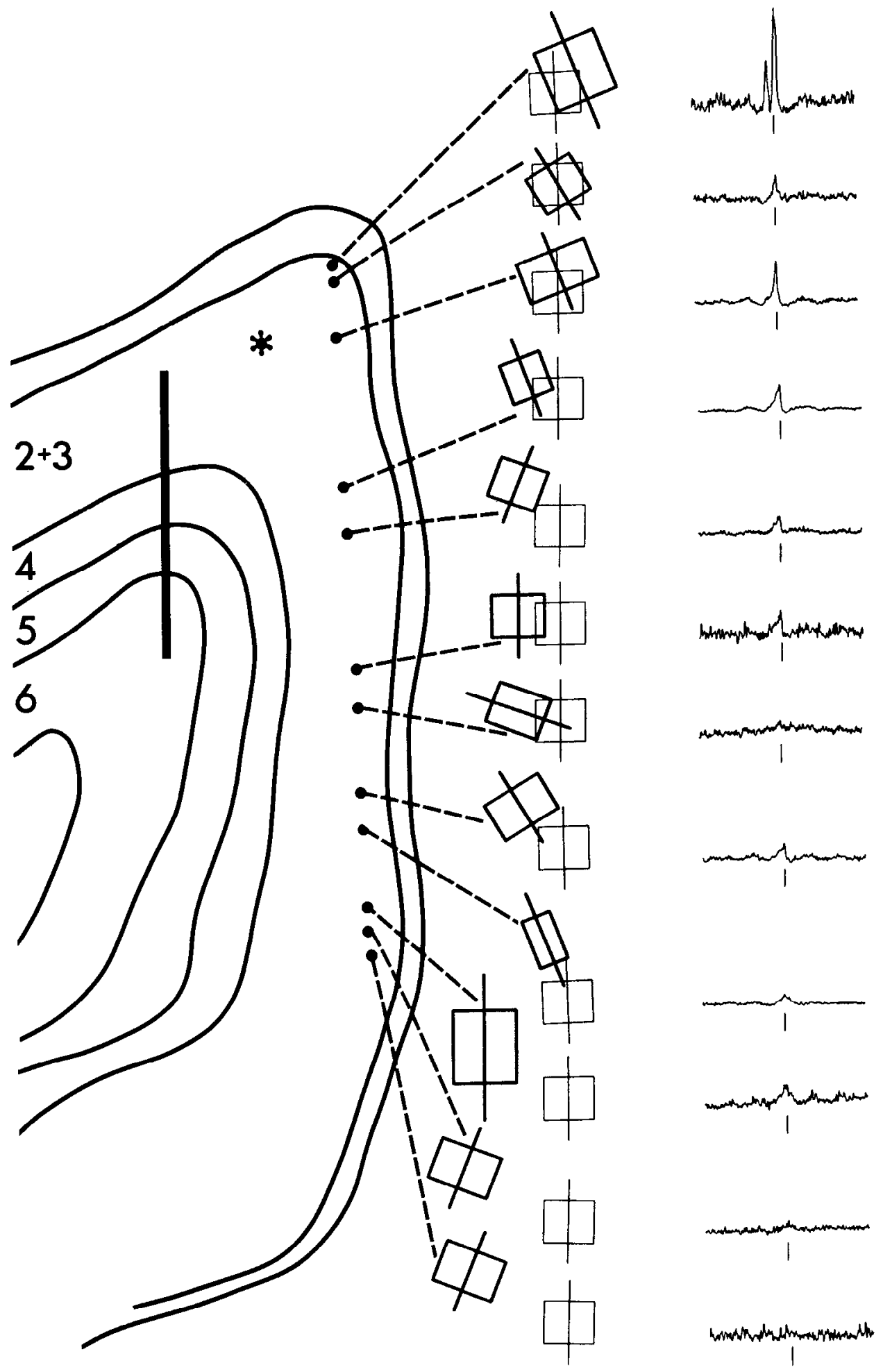

Figure 6. Medial bank penetration similar to Figure 3. Note several correlograms with monosynaptic character-peaks that lie entirely to one side of the zero time bin. correlograms, even though the receptive fields of the cells did not overlap. The last pair of receptive fields was separated by $3^{\circ}$ (center to center).

Figure 5 is another example of cross-correlograms from a penetration of a few millimeters. As in the previous penetration, peaked correlograms were found in regions where the two cells had similar receptive field properties and flat correlograms were observed when the cells had properties that differed. Figure 5 also shows that the strength of the correlogram peaks tended to diminish as the distance between the electrodes increased. The shrinking size of the peaks with distance, though not apparent in Figure 4, is more characteristic of the tangential penetrations. Typically, the interactions observed at $1 \mathrm{~mm}$ were less than half the strength of those observed in close proximity to the reference site (less than $500 \mu \mathrm{m}$ ).

A third penetration (Fig. 6), as well as the penetrations already described, had several correlograms with peaks that did not straddle the zero time bin. This pattern is characteristic of monosynaptic interactions. In particular, the first correlogram in Figure 6 had a double peak, a monosynaptic peak (on the left), and a common input peak (on the right). The monosynaptic peak accumulated in the negative time bins, indicating that the ref- 
Figure 7. Correlograms obtained from two neighboring cell pairs. One cell in each pair was the same and used as the reference cell. All threc cells had matched receptive field properties. The first test cell was separated from the reference cell by $250 \mu \mathrm{m}$. The second test cell was separated from the first test cell by $50 \mu \mathrm{m}$.

(1)

A
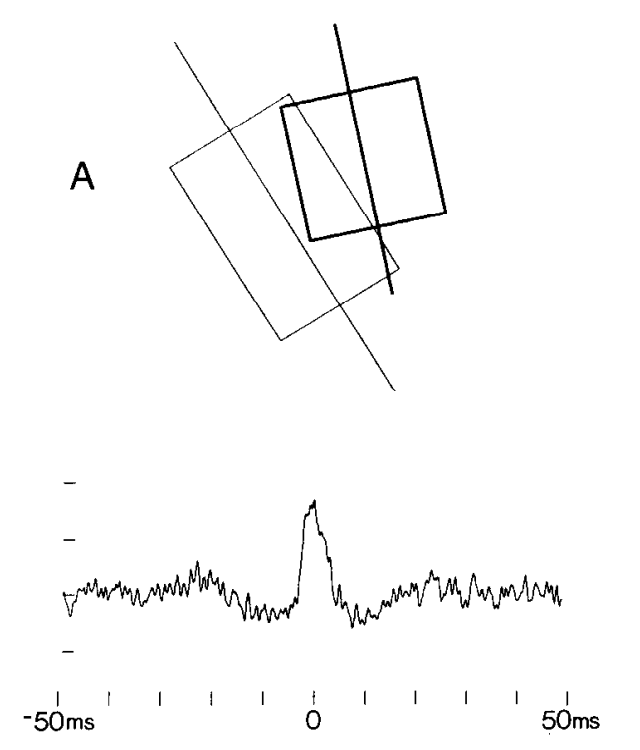
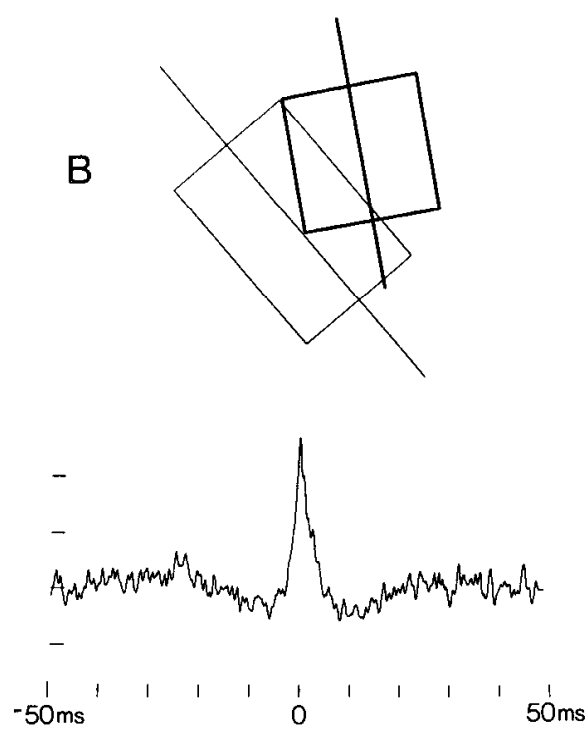

erence cell was postsynaptic to and tended to fire after the cell recorded in the second electrode. Of the peaked correlograms we obtained, $29 \%$ showed peaks characteristic of monosynaptic interactions (see first in Fig. 4). Another 20\% had peaks with an ambiguous interpretation, possibly a combination of monosynaptic and common input interactions (see sixth in Fig. 4). The remaining $51 \%$ appeared to be due to common input interactions alone (see fifth in Fig. 4).

As evident in the above penetrations, neighboring cells not only tended to have matched receptive field properties, but also similar correlograms with respect to the reference cell. This behavior is more directly illustrated in Figure 7 . Figure $7 \mathrm{~A}$ shows a peaked correlogram between two cells. One electrode was then moved $50 \mu \mathrm{m}$ to isolate another cell with similar receptive field properties, and the new pair yielded a correlogram with a comparable peak (Fig. 7B). Since the interactions of neighboring cells were similar, we were able to be less concerned about holding the same reference cell throughout the entire recording session. In situations in which it was not possible to hold the reference cell, we substituted a neighboring cell in the immediate vicinity (within $50 \mu \mathrm{m}$ ) with matching receptive field properties.

To compile our results on the relationship between the crosscorrelograms and orientation specificity, we first computed an index of the strength of the interaction between the cell pairs, as derived from the correlogram (see Materials and Methods). This strength index was calculated for the 115 pairs of cells and grouped into four bins. Figure 8 shows representative correlograms that fell in each of the four strength groups. Group 0 correlograms were flat, group 1 were either uncertain or weak, group 2 were of moderate strength, and group 3 were strong. The cell pairs were also grouped according to the difference in orientation preference between the two cells. Histograms were plotted for each of five orientation difference groups showing the relative proportion of cell pairs in each correlation strength bin (Fig. 9). For cell pairs with similar orientation tuning, e.g., $0^{\circ}-30^{\circ}$ difference, over $75 \%$ of the cell pairs yielded correlograms in groups 2 and 3 . For cell pairs with very different orientation tuning, $60^{\circ}-90^{\circ}$ difference, all $(100 \%)$ of correlogram strengths fell within groups 0 and 1 . Some (less than $25 \%$ ) cell pairs that had similar orientation preference did not exhibit significant interactions. These findings on the relationship between matching orientation preference and strength of correlation yield a $x^{2}$ significance level of $p \ll 0.01$. As would be expected from the columnar organization of the cortex, nearby cells (e.g., within $500 \mu \mathrm{m}$ ) had similar receptive field properties, which might lead to a confusion between a dependence on interelectrode distance and orientation preference. However, the relationship between orientation preference and correlation strength was observed irrespective of interelectrode distance.

Other receptive field features wcre cxamincd for thcir rclationship to the strength of correlation. We restricted the sample of cell pairs included in this analysis to cell pairs that had orientation preferences within $30^{\circ}$ and separations of at least 500 $\mu \mathrm{m}$. These parameters were chosen so as to minimize any masking cffect from the more influcntial factors: orientation preference and distance. To maximize the likelihood of detection of
0

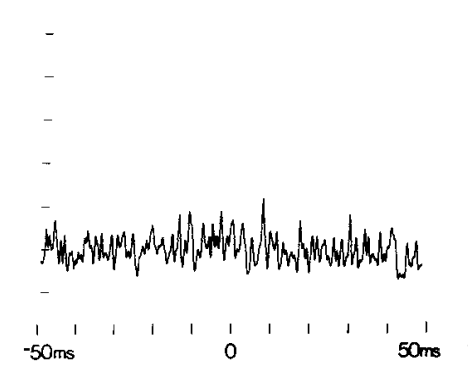

1

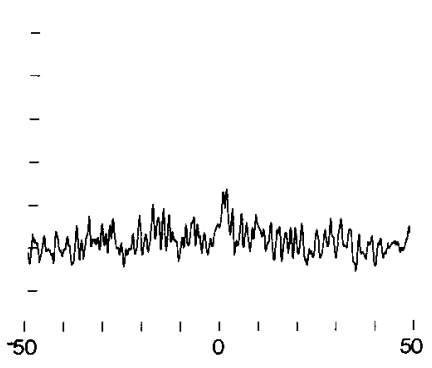

2

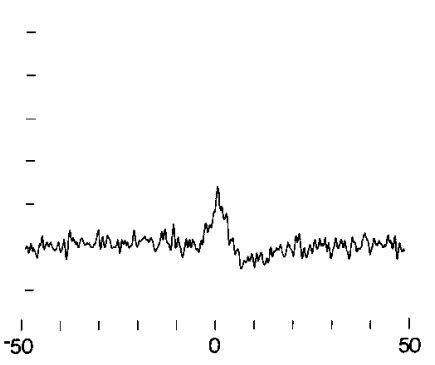

3

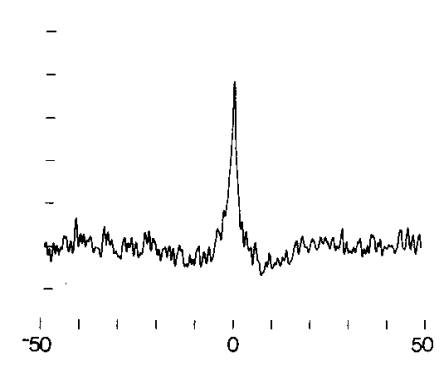

Figure 8. Correlograms from cell pairs representative of each of four correlation strength groups. Group 0 correlograms were flat; group 1 correlograms were almost flat; group 2 and 3 correlograms were moderate and strong, respectively. Placement of a cell pair in its correlation strength was performed by a computer program that estimated the energy in the peak. 


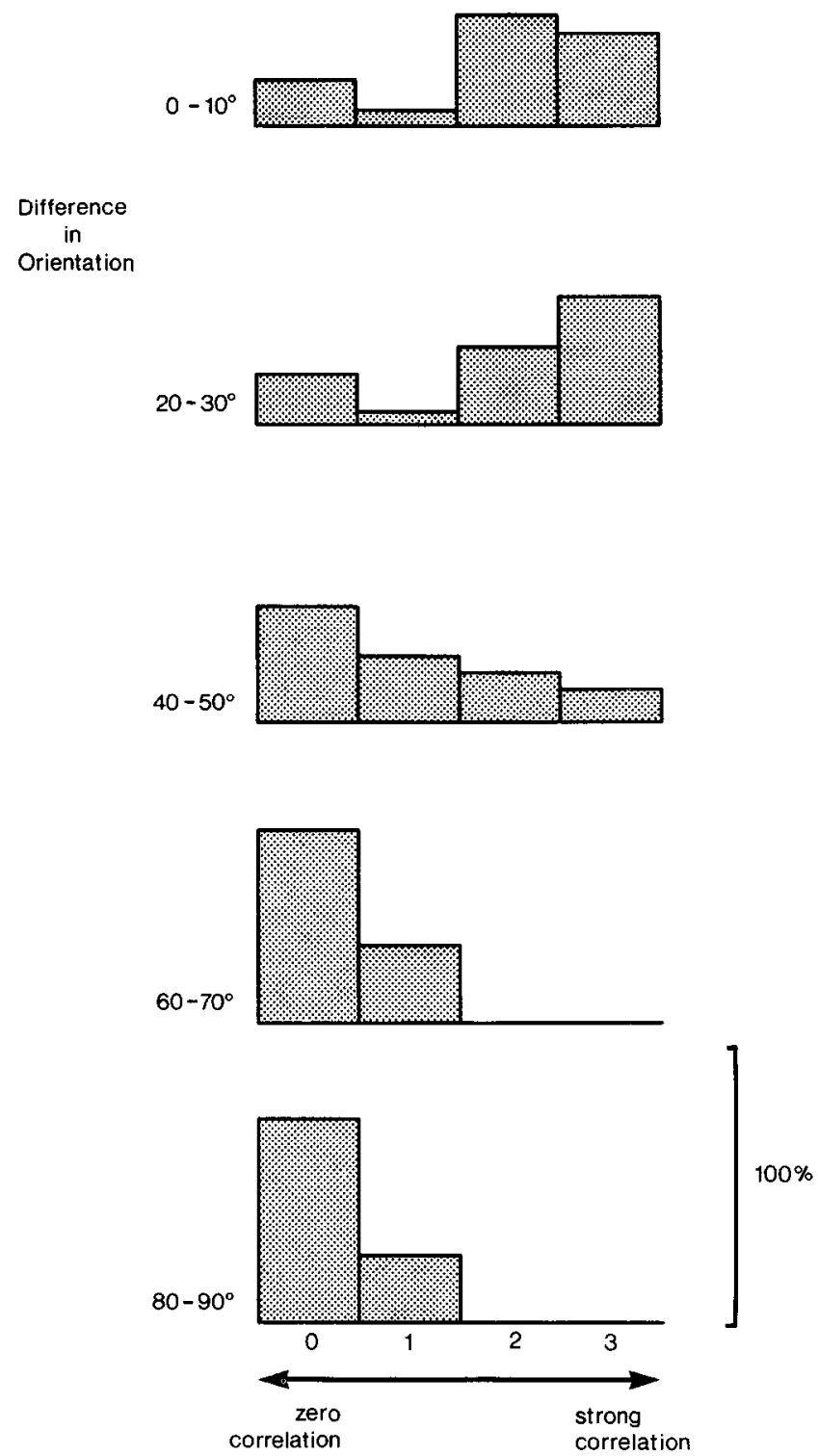

Figure 9. Histograms displaying percentage of total cell pairs falling in each of four correlation strength groups for various differences in orientation. The difference in orientation preference between the cells in each pair was computed along with its correlation strength group. Total sample: 115 cell pairs.

possible relationships, for each receptive field feature, only cell pairs classified in the strongest groups of the feature were considered (e.g., ocular dominance groups 1, 2, 6, and 7). The results for ocular dominance and directionality are summarized in $\mathrm{Ta}$ ble 1 . There was a tendency to find little or no correlation when the two cells in the pair had opposite ocular dominance, and a tendency toward a moderate or strong correlation when cells had matching ocular dominance. However, strength of correlation was much more closely related to matching orientation preference than matching ocular dominance. For directionality, on the other hand, no consistant difference was found in the strength of correlation between cells having matched or opposing directional preference. Also, we saw no significant relationship between the strength of correlation and end-inhibition, edge preferences (leading or trailing), or response to stationary flashing stimuli (ON versus OFF). However, since our resultant sample size was small, a weak relationship between the strength of correlation and the receptive field properties we measured would be undetectable. Furthermore, our analysis centered on consis-
Table 1. Relationship between ocular dominance, directionality, and strength of correlation

\begin{tabular}{clllllll} 
& & \multicolumn{5}{c}{ Correlation strength } \\
& & group & & & \\
\cline { 2 - 5 } & 0 & 1 & 2 & 3 & \\
\hline Ocular dominance & Opposite & 2 & 1 & 0 & 0 & \\
$(n=16)$ & Same & 5 & 0 & 6 & 2 & \\
Directionality & Opposite & 3 & 1 & 5 & 2 & \\
$(n=29)$ & Same & 4 & 1 & 8 & 5 & $p<0.06$
\end{tabular}

Cell pairs from the total sample ( $n=115$ ) were included if the distance between the two cells of the pair was at least $500 \mu \mathrm{m}$ and the difference in orientation preferences was within $30^{\circ}$. In addition, for ocular dominance, cell pairs were chosen where both cells fell in the ocular dominance groups $1,2,6$, or 7 . Cell pairs were classified as having opposite ocular dominance if the two cells had different eye preferences (one cell in group 1 or 2 , the other cell in 6 or 7 ). For directionality, cell pairs were chosen where both cells fell in the directionality strength groups 2,3 , or 4 . Cell pairs were classified as having opposite directionality if the two cells preferred opposing directions of stimulus motion. $\chi^{2}$ significance levels were computed grouping together correlation strengths 0 with 1 and 2 with 3 .

tent relationships among a population of cells. The absence of a consistent relationship in the population does not preclude a specific relationship between any two single cells.

Many of the correlograms had peaks that straddled the zero time bin, though sometimes asymmetrically. Such peaks are usually indicative of a significant shared input between the cells under study. Further confirmation of this shared or common input was provided in a few experiments in which the glutamate analog DLH was used as an alternative to visual stimulation to raise the firing rate of the cell pair. In separate experiments using two closely placed electrodes, we found that the effective range of the iontophoresed DLII using currents up to $10 \mathrm{nA}$ was less than $50 \mu \mathrm{m}$. In all cases the response of the recorded cell increased severalfold and recovered within $15 \mathrm{sec}$ after the application of DLH. The localization of iontophoretically applied DLH allowed us to stimulate just a small number of cells in the immediate vicinity of each of the two electrodes. If any third cell providing the common input was outside this radius, its contribution to the correlogram would be eliminated. The numbers of spikes collected in the light and DLH stimulation trials were similar so as to allow a meaningful comparison of the resultant correlograms. Figure 10 shows this comparison for a cell pair with similar receptive field properties that was separated by $320 \mu \mathrm{m}$. The lack of correlated firing under DLH iontophoresis indicates no direct synaptic connection between the two cells, but rather that the correlated firing seen with visual stimulation must be due to common input from a third cell.

Another method we used to explore the connectivity underlying peaks in the correlograms was to vary the visual stimulus. Usually, each of the two cells under study was given optimal visual stimuli, though in most cases the correlogram was unchanged with a range of orientations that excited both of the cells. An example of a correlogram that was sensitive to the orientation of the stimulus is shown in Figure 11. In this pair, it seemed possible to distinguish the orientation preference of the two recorded cells from the cells responsible for the common input. Figure 11 shows a comparison between the effect of bars of different orientations on the correlograms obtained from a pair of cells. These cells had slightly different orientation preferences. When they were stimulated with a single bar oriented between their preferred orientations, a peaked correlogram was obtained. They were then stimulated with two bars matching their preferred orientations, and a flat correlogram was obtained. An identical correlogram using the single bar of intermediate orientation was repeated as a control. Our interpretation of this finding is that the cell (or cells) providing common input pre- 
A

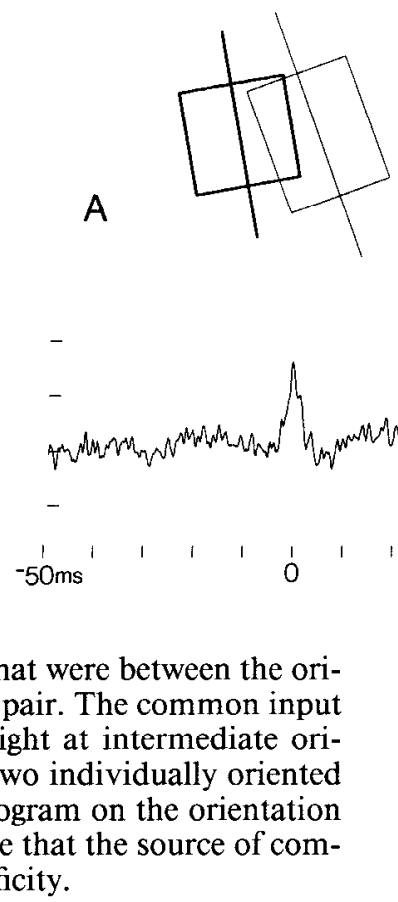

$-5$ pair with visual stimulation $(l e f t)$ and with iontophoretically applied DLH stimulation (right). The cell pair had similar receptive field propertics and were separated by $320 \mu \mathrm{m}$.
Figure 10. Correlograms from a cell

sumably had orientation preferences that were between the orientation preferences of the cells in the pair. The common input cells responded to the single bar of light at intermediate orientation but failed to respond to the two individually oriented stimuli. The dependence of the correlogram on the orientation of the light stimulus is further evidence that the source of common input itself has orientation specificity.

\section{Discussion}

The results provide a possible physiological counterpart to previous studies of the horizontal connections. The main finding of the present study was that in long electrode penetrations correlated firing with respect to a reference ccll waxcd and wancd as the electrode moved from regions of cells having matched orientation preferences to regions where the orientation was different. This arrangement is shown schematically in Figure 12. Though the strongest relationship between receptive field propertics and strength of correlation was seen for orientation, ocular dominance was also a factor. We saw no consistent relationship for the other receptive field properties tested. The observed interactions were exclusively excitatory and extended over several millimeters, including cells with fields separated by several degrees. These findings confirmed predictions that the horizontal projections of the longest extent involve cells and columns with nonoverlapping receptive fields and like orientation specificity (Gilbert and Wiesel, 1981, 1983; Mitchison and Crick, 1982). Furthermore, we believe that the distribution of correlated firing and its relationship to receptive field properties correspond to the anatomical evidence showing the clustered nature of the horizontal connections (Gilbert and Wiesel, 1979, 1983). An analogous relationship between horizontal connections and functional architecture exists in the monkey striate cortex, where the cytochrome oxidase-rich blobs are interconnected and have cells with similar receptive field properties (Livingstone and Hubel, 1984a, b).

Another measure of the strength of interaction has been termed the contribution (Levick et al., 1972). This index is defined as the percentage of the total number of spikes collected from a cell that are contained in the cross-correlogram peak. The contributions represented by our correlograms ranged from approximately 0.5 to $20 \%$ of the cells' total firing; the average was $6 \%$. At the shorter distances we studied (500 $\mu \mathrm{m}$ and less), the strength of interactions we found generally agreed with those of the previous studies of interlaminar cross-correlations by Toyama (1981 a, b). Michalski et al. (1983) also found strong direct input as well as common input interactions among cells in close proximity. However, at longer distances we observed that the strength of the interactions between columns of like specificity dropped as the horizontal distance increased and became significantly wcaker. This observation is consistent with the decrease in the extent of arborization and number of synaptic boutons in the distant clusters of HRP-injected cells (Gilbert and Wiesel, 1983). Although the observed horizontal interactions, shared and direct, between two given cells may be relatively weak, each cell is likely to receive nonshared input from many other cells distributed over a wide territory. This organization would increase the importance of the horizontal connections to a cell.

The interactions we observed were excitatory in nature, though there has been speculation concerning the existence of inhibitory horizontal connections in the cortex. It has been shown that the cross-correlation technique may be incapable of detecting weak inhibitory interactions (Aertsen and Gerstein, 1985), and, in fact, we rarely saw direct inhibitory interactions at the interelectrode distances we studied. At shorter distances, Toyama (1981a, b) and Michalski et al. (1983) did see inhibitory interactions with the cross-correlation technique. Inhibitory interactions have been seen in other systems, such as among retinal ganglion cells and auditory cortical cells (Dickson and Gerstein, 1974; Mastronarde, $1983 \mathrm{a}, \mathrm{b}$ ). Of the common input correlograms that have broad positive peaks, it is possible that some could be due to common inhibitory input (Moore et al., 1970). Nevertheless, the longest range horizontal connections are probably made by pyramidal cells, which are presumably excitatory. Presumptive
Figure 11. Correlograms from a cell pair with visual stimulation oriented differently. One cell of the pair had an orientation preference of $100^{\circ}$, and the second cell had an orientation preference of $130^{\circ}$. The two cells had matched directionality and ocular dominance and were separated by $350 \mu \mathrm{m}$. The pair was first stimulated with a single light slit oriented at $120^{\circ}(A)$, then with two slits simultaneously at $110^{\circ}$ and $150^{\circ}(B)$.
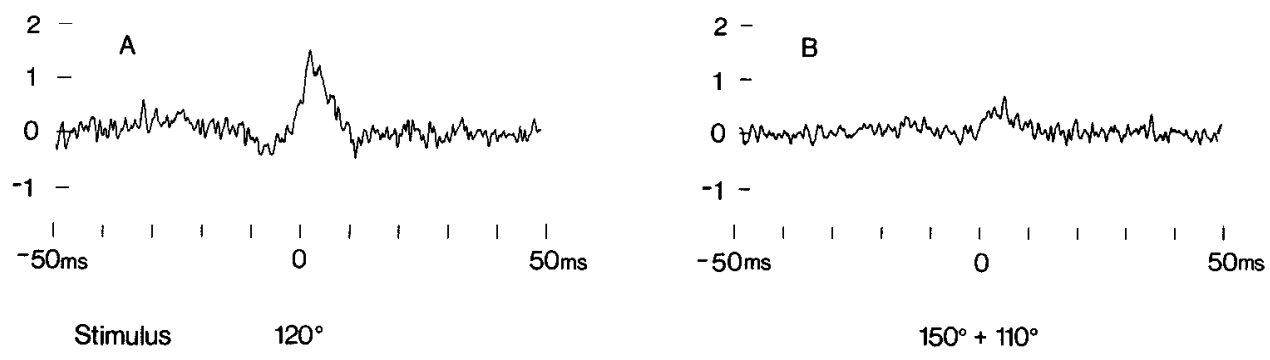


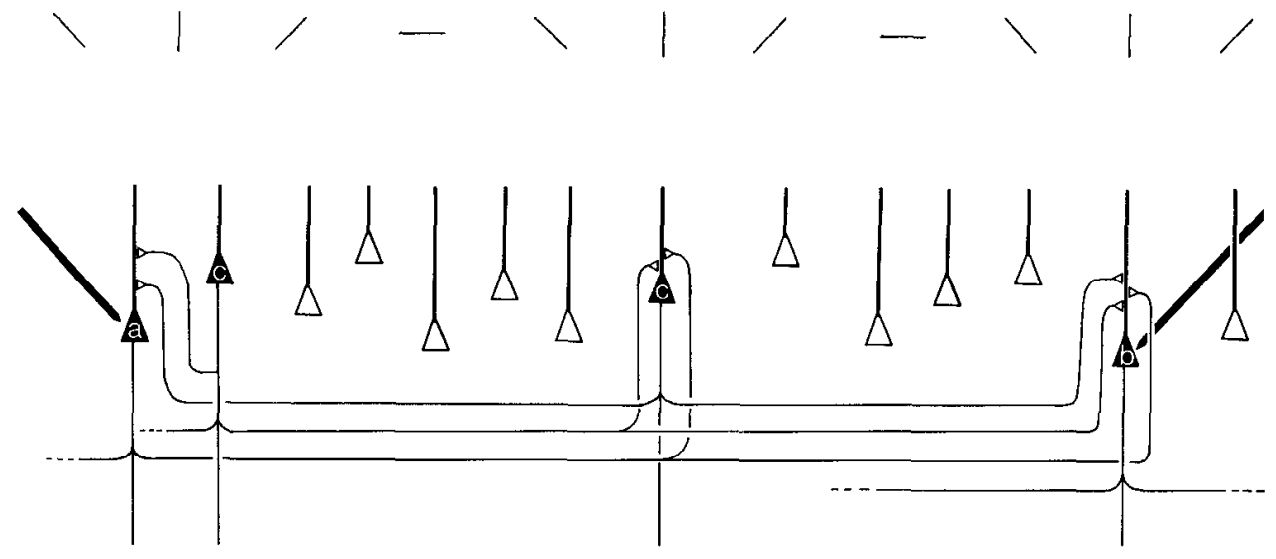

Figure 12. Schematic representing results on long-range horizontal connections betwcen columns of cclls with similar receptive field properties. Recording from the two cells $a$ and $b$, having similar orientation preferences (shown at the top), cell $a$ is seen to provide a direct input to cell $b$. In addition, the pair receives common input from several $c$ cells in cortical columns with like orientation specificity with intervening columns of differing specificity The common input connections outnumber the direct inputs from $a$ to $b$. inhibitory cells, such as the smooth stellate cells, have axons that tend to be more restricted in their horizontal extent than the distances over which we have observed correlated firing. Basket cells, also presumed to be inhibitory, do exhibit axons lengths on the order of $1 \mathrm{~mm}$ (Somogyi et al., 1983). Since we have detected correlated firing at separations of nearly $3 \mathrm{~mm}$, the underlying projections should be in that range.

There is anatomical evidence suggesting that horizontal projections are excitatory. One piece of evidence comes from experiments examining degenerating terminals of horizontal connections seen after a narrow lesion spanning all the cortical layers (Creutzfeldt et al., 1977; Fisken et al., 1975). These terminals formed asymmetric type I synapses, which are though to be excitatory. More recently, McGuire et al. (1985) studied the terminals of long-range horizontal projections of an HRP-injected cell in the monkey visual cortex and found that, in addition to forming asymmetric synapses, a majority of boutons ended on spines, presumably on spiny dendrites. Since spiny cells are though to be excitatory, these findings support the idea that horizontal connections produce excitatory influences.

Combining the labeling pattern of focal HRP injections in area 18 with a prior physiological mapping of the same tissue, Matsubara et al. (1985) concluded that connections exist between columns of orthogonal specificity and suggested that they were inhibitory. While our ability to detect weak inhibitory interactions may be in doubt, it is surprising that the excitatory connections between columns of like specificity described in the present study were not observed (although the two studies deal with different cortical areas).

Many of the cross-correlograms had peaks that were highly asymmetric but contained significant counts on both sides of the zero bin, possibly due to a combination of common input and monosynaptic excitatory interactions. Michalski et al. (1983) also found common input interactions to be more prevalent than direct interactions. In some cases where we obtained a peaked correlogram with visual stimuli, we investigated the extent of common input by stimulating each cell in the pair with the glutamate analog DLH. In most cases we found flat correlograms with DLH stimulation indicating undetectable direct connections between the two cells. We concluded that a Inajority of the positive correlations resulted from common input from cells outside the local zone of DLH excitation.

The large proportion of common input correlation at first disturbed us, but on further reflection seemed quite reasonable because of the pattern of connections observed with intracellular HRP injections. An individual cell gives off clusters of collaterals near its own dendritic field as well as at distant sites (Fig. 12). Consequently, one would expect that a given cell receives input from a group of cells that is highly interconnected. This pattern of connections would lead to common input together with direct excitation. If a large number of cells are involved in this network, the contribution of common input to the correlated firing would be relatively high. It is then expected that the strength of input shared by two cells with like specificity would exceed the strength of any direct connection they may have between each other. Correlated firing due to common input should then be more readily detectable than monosynaptic interactions.

Since we were interested in horizontal cortical connections, we needed to be certain that most or all of the correlated firing we found came from within the cortex. Correlograms with a monosynaptic component clearly represent intrinsic connections. However, the prevalence of common input correlation introduces the possibility that the common input might not be cortical but geniculate in origin. This explanation is unlikely, since the presence of correlated firing is shown to depend on matched orientation preferences. Since geniculate cells are strictly monocular and have center-surround receptive fields, common geniculate input would not be expected to provide input to cells of a specific orientation preference, but rather to cells that have the appropriate ocular dominance. In addition, reconstructions of geniculate axonal arbors have shown them to innervate a full ocular dominance column, making it unlikely that they would only contact cells of a single orientation (Blasdel and Lund, 1983; Ferster and LeVay, 1978; Gilbert and Wiesel, 1983; Hubel and Wiesel, 1972). Another piece of evidence is that on a number of occasions we found that the shape of the correlogram between a particular cell pair was dependent on the orientation of the stimulus, indicating that the source of common input to the cells was itself oriented, and thus unlikely to be geniculate in origin (Fig. 10). Other possible sources of common input are the recurrent projections from extrastriate cortex which also have a clustered distribution (Gilbert and Kelly, 1975; Wong-Riley, 1979). Given that many of the interactions we observed had a monosynaptic component resulting from intrinsic connections, these connections must contribute to common input interactions as well. It is possible that both corticocortical connections and intrinsic connections contribute to the interactions between columns of like specificity.

There are a number of functional properties of visual cortical neurons that may involve horizontal interactions. Inhibitory interactions between orientation columns of differing specificity may sharpen orientation tuning (Blakemore and Tobin, 1972; Kemp et al., 1981; Sillito, 1977). However, the extent to which such interactions exist has been questioned (Ferster, 1984; Wolf et al., 1983). In any event, to sharpen orientation tuning one would expect interactions between cells of differing orientations with overlapping receptive fields, whereas the interactions shown by either the anatomical connections or by the present crosscorrelation study can involve cells with widely separated receptive fields. Conceivably, long-range interactions could produce 
inhibitory flanks beyond the excitatory portion of a receptive field, producing the properties of end-inhibition and side-band inhibition (Gilbert and Wiesel, 1983). However, since they are facilitatory, the interactions we have studied are unlikely to be related to the above properties. The extent of the horizontal connections and their facilitatory nature suggest that they may contribute to properties beyond those determined by classical receptive field analysis (Allman et al. 1985).

\section{References}

Aertsen, A., and G. L. Gerstein (1985) Evaluation of neuronal connectivity: Sensitivity of cross-correlation. Brain Res. 340: 341-354.

Allman, J., F. Miezin, and E. McGuinness (1985) Stimulus specific responses from beyond the classical receptive field: Neurophysiological mechanisms for local-global comparisons in visual neurons. Annu. Rev. Neurosci. 8: 407-430.

Blakemore, C., and E. Tobin (1972) Lateral inhibition between orientation detectors in the cat's visual cortex. Exp. Brain Res. 15: 439440.

Blasdel, G. G., and J. S. Lund (1983) Termination of afferent axons in macaque striate. J. Neurosci. 3: 1389-1413.

Bryant, H. L., Jr., A. Ruiz Marcos, and J. P. Segundo (1972) Correlations of neuronal spike discharges produced by monosynaptic connections and by common inputs. J. Neurophysiol. 35: 864-878.

Creutzfeldt, O. D., L. J. Garey, R. Kuroda, and J. R. Wolff (1977) The distribution of degenerating axons after small lesions in the intact and isolated visual cortex of the cat. Exp. Brain Res. 27: 419-440.

Dickson, J. W., and G. L. Gerstein (1974) Interactions between neurons in auditory cortex of the cat. J. Neurophysiol. 37: 1239-1261.

Ferster, D. (1984) Receptive field properties of EPSPs and IPSPs in cat visual cortex. Soc. Neurosci. Abstr. 10: 512.

Ferster, D., and S. LeVay (1978) The axonal arborization of lateral geniculate neurons in the striate cortex of the cat. J. Comp. Neurol. 182: 923-944.

Fisken, R. A., L. J. Garey, and T. P. S. Powell (1975) The intrinsic, association and commissural connections of area 17 of the visual cortex. Phil. Trans. R. Soc. 272: 487-536.

Gilbert, C. D., and J. P. Kelly (1975) The projections of cells in different layers of the cat's visual cortex. J. Comp. Neurol. 163: 81106.

Gilbert, C. D., and T. N. Wiesel (1979) Morphology and intracortical projections of functionally identified neurons in cat visual cortex. Nature 280: 120-125.

Gilbert, C. D., and T. N. Wiesel (1981) Laminar specialization and intracortical connections in cat primary visual cortex. In The Organization of the Cerebral Cortex, F. O. Schmitt, F. G. Worden, G. A. Adelman, and S. G. Dennis, eds., pp. 163-191, MIT Press, Cambridge, MA.

Gilbert, C. D., and T. N. Wiesel (1983) Clustered intrinsic connections in cat visual cortex. J. Neurosci. 3: 1116-1133.

Gustafsson, B., and D. McCrea (1984) Influence of stretch-evoked synaptic potentials on firing probability of cat spinal motoneurones. J. Physiol. (Lond.) 347: 431-451.

Hubel, D. H. (1957) Tungsten microelectrodes for recording from single units. Science 125: 549-550.

Hubel, D. H., and T. N. Wiesel (1962) Receptive fields, binocular interaction and functional architecture in the cat's visual cortex. $\mathbf{J}$. Physiol. (Lond.) 160: 106-154.

Hubel, D. H., and T. N. Wiesel (1972) Laminar and columnar distribution of geniculocortical fibers in the macaque monkcy. J. Comp. Neurol. 146: 421-450.

Hubel, D. H., and T. N. Wiesel (1974) Uniformity of monkey striate cortex: A parallel relationship between field size, scatter, and magnification factor. J. Comp. Neurol. 158: 295-306.

Kasser, R. J., and P. D. Cheney (1983) Double-barreled electrode for simultaneous iontophoresis and single-unit recording during movement in awake monkeys. J. Neurosci. Methods 7: 235-242.

Kemp, J. A., P. C. Murphy, and A. M. Sillito (1981) Cholinergic influences on the response properties of cells in the cat visual cortex. J. Physiol. (Lond.) 320: 16-17.

Kirkwood, P. A. (1979) On the use and interpretation of cross-cor- relation measurements in the mammalian central nervous system. J. Neurosci. Methods 1: 107-132.

Levick, W. R., B. G. Cleland, and M. W. Dubin (1972) Lateral geniculate neurons of cat: Retinal inputs and physiology. Invest. Ophthalmol. 11: 302-311.

Livingstone, M. S., and D. H. Hubel (1984a) Anatomy and physiology of a color system in the primate visual cortex. J. Neurosci. 4: 309356.

Livingstone, M. S., and D. H. Hubel (1984b) Specificity of intrinsic connections in primate primary visual cortex. J. Neurosci. 4: 28302835.

Lorente de No, R. (1933) Studies on the structure of the cerebral cortex. J. Psychol. Neur. 45: 382-438.

Martin, K. A. C., and D. Whitteridge (1984) Form, function and intracortical projections of spiny neurones in the striate visual cortex of the cat. J. Physiol. (Lond.) 353: 463-504.

Mastronarde, D. N. (1983a) Correlated firing of cat retinal ganglion cells. I. Spontaneously active inputs to $\mathrm{X}$ - and Y-cells. J. Neurophysiol. 49: 303-324.

Mastronarde, D. N. (1983b) Correlated firing of cat retinal ganglion cells. II. Responses of X- and Y-cells to single quantal events. J. Neurophysiol. 49: 325-349.

Matsubara, J., M. Cynader, N. V. Swindale, and M. P. Stryker (1985) Intrinsic projections within visual cortex: Evidence for orientationspecific local connections. Proc. Natl. Acad. Sci. USA 82: 935-939.

McGuire, B. A., C. D. Gilbert, and T. N. Wiesel (in press) Ultrastructural characterization of long-range clustered horizontal connections in monkey striate cortex. Soc. Neurosci. Abstr.

Michalski, A., G. L. Gerstein, J. Czarkowska, and R. Tarnecki (1983) Interactions between cat striate cortex neurons. Exp. Brain Res. 51: 97-107.

Mitchison, G., and F. Crick (1982) Long axons within the striate cortex: their distribution, orientation and patterns of connection. Proc. Nat. Acad. Sci. USA 79: 3661-3665.

Moore, G. P., D. H. Perkel, and J. P. Segundo (1970) Statistical signs of synaptic interaction in neurons. Biophys. J. 10:876-900.

Otsuka, R., and R. Hassler (1962) Über Aufbrau und Gliederung der corticalen Sehsphare bei de Katz. Arch. Psychiatr. Nerven. 203: $212-$ 234.

Perkel, D. H., G. L. Gerstein, and G. P. Moore (1967a) Neuronal spike trains and stochastic point processes. I. The single spike train. Biophys. J. 7: 391-418.

Perkel, D. H., G. L. Gerstein, and G. P. Moore (1967b) Neuronal spike trains and stochastic point processes. II. Simultaneous spike trains. Biophys. J. 7: 419-440.

Rockland, K. S., and J. S. Lund (1983) Intrinsic laminar lattice connections in primate visual cortex. J. Comp. Neurol. 216: 303-318.

Rockland, K. S., J. S. Lund, and A. L. Humphrey (1982) Anatomical banding of intrinsic connections in striate cortex of tree shrews ( $T u$ paia glis). J. Comp. Ncurol. 209: 41-58.

Sillito, A. M. (1977) Inhibitory processes underlying the directional specificity of simple, complex and hypercomplex cells in the cat's visual cortex. J. Physiol. (Lond.) 289: 33-35.

Somogyi, P., Z. F. Kisvarday, K. A. C. Martin, and D. Whitteridge (1983) Synaptic connections of morphologically identified and physiologically characterized large basket cells in the striate cortex of cat. Neuroscience 10: 261-294.

Surmeier, D. J., and R. J. Weinberg (1985) The relationship between cross-correlation measures and underlying synaptic events. Brain Res. 331: $180-184$.

Toyama, K., M. Kimura, and K. Tanaka (1981a) Cross-correlation analysis of interneuronal connectivity in cat visual cortex. J. Neurophysiol. 46: 191-201.

Toyama, K., M. Kimura, and K. Tanaka (1981b) Organization of cat visual cortex as investigated by cross-correlation techniques. J. Neurophysiol. 46: 202-214.

Wolf, W., K. Albus, and T. P. Hicks (1983) Effects of blocking GABAergic inhibition on visual response properties of neurones in the kitten's striate cortex. Neurosci. Lett. Suppl. 14: S407.

Wong-Riley, M. (1979) Columnar cortico-cortical interconnections within the visual system of the squirrel and macaque monkeys. Brain Res. 162: 201-217. 